\title{
Inequality and Conservation on the Local Commons: A Theoretical Exercise

\author{
JefF Dayton-Johnson ${ }^{1}$ and Pranab Bardhan ${ }^{2}$
}

August 1999

\begin{abstract}
To analyze the effect of asset inequality on cooperation within a group, we consider a two-player noncooperative model of conservation of a commonpool resource. Overexploitation by one user affects another's payoff by reducing the next-period catch. We give necessary and sufficient conditions such that conservation is a Nash equilibrium, and show that increasing inequality does not, in general, favor full conservation. However, once inequality is sufficiently great, further inequality can raise efficiency. Thus the relationship between inequality and economic efficiency is U-shaped. Finally, we analyze the implications for conservation if players have earning opportunities outside the commons.
\end{abstract}

keywords: collective action, common property, exit options, inequality

JEL classification codes: D63, D71, H41, O12, O13, Q22, Q25

\footnotetext{
${ }^{1}$ Corresponding author. Department of Economics, Dalhousie University, Halifax, Nova Scotia, CANADA B3H 3J5; (902) 494-2026 (telephone), (902) 494-6917 (fax), jeff.dayton-johnson@dal.ca (Internet).

${ }^{2}$ Department of Economics, University of California, Berkeley, 549 Evans Hall \# 3880, Berkeley, CA 94720-3880 USA.
} 


\section{INTRODUCTION}

1.1. The commons dilemma and inequality. The daily livelihood of vast masses of the rural poor in many countries depends on the success with which common pool resources (CPRs) - such as forest resources, grazing lands, in-shore fisheries, and irrigation water - are managed, and on the environmental consequences of their management. A CPR is defined by Ostrom (1990, p. 30) as "a natural or man-made resource system that is sufficiently large as to make it costly (but not impossible) to exclude potential beneficiaries from obtaining benefits from its use." Understanding the factors that lead to success or failure of community management of these resources is critical to rural development.

CPR management is a collective-action dilemma: a situation in which mutual cooperation is collectively rational for the group as a whole, but individual cooperation is not necessarily individually rational for each member. One factor that has not always been recognized as critical to the outcome of collective action dilemmas is heterogeneity among the resource users. Ostrom (1990), whose analysis of local management of CPRs is probably the best-known, discusses homogeneity and heterogeneity, but excludes the issue from her list of factors crucial to successful CPR management. In this paper, our attention will be largely restricted to a single but potent kind of heterogeneity: inequality in asset ownership.

Olson (1965, p. 34) hypothesized that inequality might favor the provision of a public good:

In smaller groups marked by considerable degrees of inequality - that is, in groups of members of unequal "size" or extent of interest in the collective good - there is the greatest likelihood that a collective good will be provided; for the greater the interest in the collective good of any single member, the greater the likelihood that that member will get such a significant proportion of the total benefit from the collective good that he will gain from seeing that the good is provided, even if he has to pay all of the cost himself.

Inequality in this context can thus facilitate the provision of the collective good, with the small players free-riding on the contribution of the large player. In a very general setting, Bergstrom, Blume and Varian (1986) show that wealth redistributions that increase the wealth of equilibrium contributors to a public good will increase the supply of the public 
good. ${ }^{3}$ These analyses of the supply of public goods are relevant to conservation among CPR users; restraint in resource use is analytically equivalent to contributing to a public good. Following these studies, we would expect group heterogeneity to be conducive to the effective local management of CPRs.

Nevertheless, field studies of CPR management have repeatedly shown that inequality is harmful for collective action. Bardhan (1995) reviews the case-study literature regarding the relationship between inequality and cooperation in locally-managed irrigation systems, primarily in Asia. Baland and Platteau $(1996,1997,1998)$ likewise summarize many relevant examples from the case-study literature; they focus more on forests, fisheries and grazing lands, and on African cases. Johnson and Libecap's (1982) study of the Texas shrimp fishery is a well-known example. The fishery is exploited by a large fleet of fishers differentiated by ability. The problem is one of overfishing: in this case of increased effort and declining per-boat yields, there exists a collective incentive to self-regulate effort, in the form of production quotas, and thereby increase collective rents. The success of such contracting, however, is conditioned by the existence of high transaction costs. Johnson and Libecap assume that side-payments are impossible to administer, and that the information costs (and presumably enforcement costs) underlying agent-specific quotas are too high to make such a quota system practical; the only option, therefore, is a system of uniform quotas. The per-agent gain in moving from the unregulated equilibrium to the rent-maximizing output level (under uniform quotas) is the same for all agents, but the per-agent loss is increasing in agents' productivity. Thus it is possible that more productive fishers stand to lose under a cooperative regime, and hence they will oppose it.

A small number of papers has sought to formalize the logic of the Johnson and Libecap example. Kanbur (1991) describes a two-person, simultaneous-move, numerical example in which the players have different payoff functions. As payoff inequality is increased, the efficient outcome ceases to be a self-enforcing equilibrium. Baland and Platteau (1997)

\footnotetext{
${ }^{3}$ Chan, Mestelman, Moir and Muller (1996) report that when the Bergstrom-Blume-Varian model is tested in the laboratory, it correctly predicts the direction (though not the magnitude) of changes in group contributions when income is redistributed toward positive contributors. It does not do so well in predicting individual behavior: individuals with low incomes overcontribute to the public good, and high-income individuals undercontribute, relative to the model.
} 
argue that inequality in resource-use entitlements is associated with higher conservation in some examples where the resource-use technology (e.g., fishing, harvesting, gathering) exhibits decreasing returns to effort. Under alternative cases featuring increasing returns to effort, the effect of increasing inequality is indeterminate.

This paper presents a model of heterogeneous resource users in a local commons dilemma. The model is couched in terms of a fishery; thus the players are referred to as fishers and the resource as the fish stock. Indeed, the crisis of conservation of fisheries globally (see, e.g., Safina 1995) is at least as advanced as resource degradation in other CPRs. In particular, fisheries are important to peasant economies throughout the developing world. Nevertheless, we chose the fishery example primarily to lend concreteness to the discussion. We hope that the basic conclusions of the model will be transferable to other CPR examples, such as groundwater-based irrigation, community grazing lands, and village forests. It should further be noted that the externality between fishers in our model differs from the usual congestion externality posited in research on the commons. In our model, one fisher's overexploitation affects another's incentives through payoffs in the following period, while the conventional congestion externality acts through increased harvesting costs during this period. In Section 4.1, nevertheless, we consider a congestion externality in a variant of our basic model.

Additionally, many researchers have argued that the commons dilemma is analogous to other social problems. Keohane and Ostrom (1994) point out that many issues in international relations bear a striking formal resemblance to CPRs: in both cases, agents have little recourse to external authority to impose efficient solutions. Others have argued that the problem of economic growth in the presence of poor property-rights protection is like a commons problem in which agents must "conserve" by forgoing current consumption of the commonly-held capital stock, or by diverting resources to the creation of institutions that protect property rights (Benhabib and Rustichini 1996; Tornell and Velasco 1992). In a similar vein, a 1999 report of the Canadian Standing Senate Committee on Social Affairs, Science and Technology identifies "social cohesion" as the "ultimate common property resource. We can all benefit from it if it exists, but it is far too easy to let the social fabric deteriorate as we each pursue our own short-term self-interest" (Government of Canada, 
1999). In cross-country regression analysis for 29 market economies, Knack and Keefer (1997) find that social cohesion (as measured by the level of trust and the propensity of people to join voluntary organizations) is strongly associated with higher per capita income growth; furthermore they find that the level of social cohesion is strongly and negatively associated with income inequality. If indeed social cohesion is a common-pool resource, then our research provides theoretical support for the negative effect of inequality detected by Knack and Keefer, although our results predict that this relationship is not strictly monotonic.

1.2. Outline of the paper. In the paper, we demonstrate that Olson's (1965) hypothesis - interpreted as a comparative-static statement that increasing inequality enhances efficiency - is not always valid. In many settings increased inequality leads to less efficiency; this is true whether or not fishers have earning opportunities outside the commons. If these exit options are concave functions of wealth, increased inequality does not, in general, enhance the prospects for full conservation. Neither is it true that perfect equality always favors greater efficiency. At a certain wealth distribution, increasing wealth inequality increases equilibrium efficiency (though not attaining full conservation as long as both fishers have positive wealth), and furthermore, full conservation is an equilibrium under perfect inequality. In the model where fishers have exit options, full conservation cannot be an equilibrium under perfect equality if average wealth is below some threshold level. (In a one-period variant of our model with myopic players and a congestion externality, we show that increasing inequality of asset ownership reduces aggregate catch.)

The related assertion that the larger player has a greater interest in collective action than the smaller is borne out in many settings: with or without concave exit options, it is the poor who do not conserve. This too is dependent on the assumptions made: if exit-option functions are convex, for example, it is the poorer fisher who has an interest in conditional conservation, while the richer fisher prefers the exit strategy. That the larger player, in many settings, has a greater interest in collective action than the smaller does not imply, however, that a more unequal distribution of wealth will lead to more successful collective action.

A significant result is that the relationship between inequality and collective action is 
not necessarily monotonic. In fact, the relationship is U-shaped: at very low and very high levels of inequality, conservation is possible, while for some middle range of inequality it is not.

The outline of the chapter is as follows. Section 2 sketches a basic noncooperative commons game. We extend the basic game in Section 3 to consider the effects on conservation if players have earning opportunities outside the commons. The two-player model we use in this chapter contributes to the tractability of the analysis and the transparency of the results. A two-player model abstracts from the group-size problem highlighted by Olson (1965) in order to better focus on the problem of inequality. In Section 4.2, the results of the basic model are extended to the many-fisher case. Section 4.1 generalizes the catch technology and cost function of the basic model. Section 5 briefly considers schemes for community regulation of the commons in light of the noncooperative model, and concludes.

\section{A Simple MODEL OF THE COMMONS}

There are two fishers, $i=1,2$. Each is endowed with wealth $e_{i}$ in each period. They share access to a common resource, namely a stock of fish $F$. In each of two periods $t$, each fisher must choose to spend some portion of his endowment on fishing capacity $a_{i}^{t}$; thus $a_{i}^{t} \leq e_{i}$. ( $a$ is short for "action.") Each fisher's utility is simply the total amount of fish he catches:

$$
U_{i}=\phi_{i}^{1}\left(a_{i}^{1}\right)+\phi_{i}^{2}\left(a_{i}^{1}\right)
$$

where $\phi_{i}^{t}(\cdot)$ is the amount of fish caught by fisher $i$ in period $t$. Fishing yield is a function $f$ of capacity deployed: $f\left(a_{i}^{t}\right)=a_{i}^{t}$ unless total capacity deployed exceeds the available fish, in which case each fisher gets a share of the total equal to his share of total wealth. (This is the situation known as "overcapitalization" in the literature on fisheries.) Each fisher's payoff in period 1 , then, is given by

$$
\phi_{i}^{1}\left(a_{i}^{1}\right)= \begin{cases}a_{i}^{1}, & a_{1}^{1}+a_{2}^{1} \leq F \\ \frac{a_{i}^{1}}{a_{1}^{1}+a_{2}^{1}} F, & a_{1}^{1}+a_{2}^{1}>F\end{cases}
$$

Between periods the stock of fish grows at rate $g$, so that in period 2 the supply of fish is $G \cdot\left(F-\phi_{1}^{1}-\phi_{2}^{1}\right)$, where $G \equiv 1+g$. In the second period, each fisher again chooses a capacity level $a_{i}^{2}$. The nature of each fisher's endowment is such that any proportion of it 
can be used in each period for fishing. It is not spent. It reflects fishing "effort", including number of boats and hours and intensity of labor. Note that in any efficient outcome there will be no fishing in period 1 . We make the following "commons dilemma assumption":

$$
E \geq G F
$$

where $E \equiv e_{1}+e_{2}$. Assumption (1) ensures that the threat of resource degradation is sufficiently acute. Alternatively, (1) can be interpreted as a "feasibility" condition: the fishers are capable of harvesting the entire stock if they desire.

In the subgame consisting of the second period, both fishers will always fish to capacity. That is, each will choose $a_{i}^{2}=e_{i}$ and receive second-period payoff

$$
\phi_{i}^{2}=\frac{e_{i}}{E} G\left(F-\phi_{1}^{1}-\phi_{2}^{1}\right)
$$

Thus we can concentrate on the fishers' actions in the first period. A strategy is just a capacity choice $a_{i}^{1}$, and the first-best outcome is $a_{1}^{1}=a_{2}^{1}=0$. Any Nash equilibrium of the abbreviated first-period game will be a subgame-perfect equilibrium of the two-period game, assuming full depletion occurs in the second period. For simplicity, we will hereafter suppress the period superscript, since all strategic choices under consideration are made in period 1. (If $G$ were less than 1, there would be no real dilemma: first-period depletion of the resource would be an equilibrium and an optimum.) The crowding externality that is sometimes a feature of commons models does not occur in our model within periods. That is, $j$ 's action in period 1 does not enter $i$ 's payoff in that period, although $j$ 's period-1 action will enter $i$ 's period-2 payoff, and vice-versa. (In Section 4.1 below, we consider a within-period congestion externality in the context of our model.)

The goal of conservation in fisheries is to reduce fishing to some level so that the remaining stock at the end of every period is sufficient to guarantee the survival of the fish population. In our simple model, that level has been normalized to zero in the first period. The second period extends to the end of the fishers' relevant economic horizons. The two-period set-up precludes consideration of complicated punishment strategies, but it is sufficient to capture the fundamental dilemma of resource conservation: namely, when is it reasonable to forgo current-period consumption in return for higher next-period gains? ${ }^{4}$

\footnotetext{
${ }^{4}$ Other economic treatments of the fishery have focused on changes in the incentives to conserve when the
} 
In this model, we have abstracted from the problem of discount rates in order to focus more clearly on the incentives toward resource conservation. Formally, the discount rate would be subtracted from $G$, the rate of fish-stock regeneration. If the discount rate is greater than $G$, first-period depletion of the fishery is optimal, and conservation is not economically rational. Furthermore, each fisher's discount rate is plausibly a decreasing function of wealth. In this case, the more unequal the distribution of endowments, the more difficult it will be to sustain universal conservation of the resource. It is as if the poor fisher faces a low rate of growth in the stock and hence has little incentive to conserve.

The following proposition notes the conditions under which the least efficient outcome is a Nash equilibrium.

Proposition 1. If $e_{i}>F(G-1) / G$ for $i=1,2$, then $\left\{e_{1}, e_{2}\right\}$ is a Nash equilibrium.

(The proof of this and all other propositions is provided in the Appendix.) In particular, note that Proposition 1 implies that if $e_{i}>F$ for $i=1,2$ - if each fisher could unilaterally harvest the entire fish stock in the first period - then complete resource depletion is an equilibrium. When is full conservation a Nash equilibrium? Proposition 2 gives necessary and sufficient conditions.

Proposition 2. In the basic game described above, when both players have positive wealth, $a_{1}=a_{2}=0$ is a Nash equilibrium if and only if $e_{i} \geq E / G$ for $i=1,2$.

The conditions of Propositions 1 and 2 can be satisfied simultaneously. In fact, whenever the condition of Proposition 2 is satisfied (i.e., $e_{i} \geq E / G, \forall i$ ), there are multiple equilibria, since the condition of Proposition 1 (i.e., $e_{i} \geq F(G-1) / G, \forall i$ ) will also be satisfied under the commons dilemma assumption (1). This is illustrated in Figure $1 . .^{5}$ The $45^{\circ}$ line shows all possible distributions of wealth $e_{1}+e_{2}=E$. Both full conservation (Proposition 2) and fish population varies (Levhari and Mirman 1980; Dutta and Sundaram 1993). This can be approximated in our model by simply varying $F$ as a comparative-static exercise.

${ }^{5}$ This figure was suggested by Timothy Besley. 
full depletion (Proposition 1) are equilibria for the range of wealth distributions $B C$. At a point such as $A$, full conservation is not an equilibrium; after an equalizing redistribution of wealth to a point between $B$ and $C$, full conservation would be an equilibrium.

\section{[Figure 1 here]}

Intuitively, $E / G$ is the threshold amount of wealth above which the fisher will conserve, conditional on his counterpart's conservation. Alternatively, the condition $e_{i} \geq E / G, \forall i$ can be interpreted as defining a minimal regeneration rate $G$ such that mutual conservation is possible in equilibrium. For the two-fisher case we are considering here, this condition is equivalent to $G \geq 2$. This means that the fish stock must grow at a rate of $100 \%$. This might seem worrisomely high; the astute reader will have moreover noticed that the $n$-player version of Proposition 2 will imply that conservation requires $G \geq n$. This result is quite robust. For any general fishing technology $f\left(a_{i}\right)$ and sharing rule $\left.\left(\left\{\alpha_{i}\right\}_{i \in I}, \sum \alpha_{i}=1\right\}\right)$ in the case of overcapitalization, it can be shown that the appropriate generalization of Proposition 2 implies that $G \geq n$, where $n$ is the number of players in the set $I$. This is not necessarily the case if the share $\alpha_{i}$ accruing to fisher $i$ is a function of first-period conservation, as it might be in the case of a regulated fishery. We will return to this point in Section 5.

This proposition suggests the following corollary. Define

$$
\Delta(E) \equiv\left\{\left(e_{1}, e_{2}\right) \mid e_{1} \geq 0, e_{2} \geq 0, e_{1}+e_{2}=E\right\}
$$

as the set of all distributions of $E$. For any $e=\left(e_{1}, e_{2}\right) \in \Delta(E), \hat{e} \in \Delta(E)$ is a meanpreserving spread of $e$ if $\left|\hat{e}_{1}-\hat{e}_{2}\right|>\left|e_{1}-e_{2}\right|$.

Corollary 1. (a) Consider $e, e^{\prime} \in \Delta(E)$, where $e^{\prime}$ is a mean-preserving spread of $e$. Then $a_{1}=a_{2}=0$ is an equilibrium under $e^{\prime}$ only if it is an equilibrium under $e$. (b) For all $e \in \Delta(E)$ there is a mean-preserving spread $e^{\prime}$ such that $a_{1}=a_{2}=0$ is not an equilibrium under $e^{\prime}$.

The Olson hypothesis that inequality enhances the prospects for collective action can be interpreted as a comparative-static statement: namely, that increasing inequality (for a 
given level of aggregate wealth) makes full conservation more likely. Corollary 1 suggests that this is not so. Part (b) states that, starting from any wealth distribution, there exists a less equal wealth distribution such that full conservation is not an equilibrium. In particular, if full conservation is an equilibrium under the initial distribution, then we know from Proposition 2 that $e_{i} \geq E / G$ for $i=1,2$. Then wealth can be taken from one fisher until $e_{i}<E / G$ for that fisher; full conservation is no longer an equilibrium. In terms of Figure 1, this is equivalent to moving from a point in the region $B C$ to a point such as $A$.

Corollary 1 illustrates that increased inequality does not necessarily lead to equilibrium conservation. Proposition 3, however, shows that under maximum inequality - that is, when one fisher owns all the wealth - conservation is an equilibrium outcome.

Proposition 3. In the basic game, if $G$ is greater than or equal to one, then under perfect inequality $(e=(E, 0)$ or $e=(0, E))$, full conservation is a Nash equilibrium.

In part, Proposition 3 reflects Olson's hypothesis that cooperation is more difficult in a group the larger the number of group members. In our fishery, conservation is an equilibrium outcome when the number of fishers with positive wealth is reduced to one.

The propositions above consider only the conditions under which full conservation by both fishers is an equilibrium outcome. The more realistic case in an unregulated fishery, and one perhaps closer to Olson's thinking, is one in which changes in the distribution of wealth change the level of efficiency among a set of inefficient equilibria. This is considered in the following proposition, which says that if the distribution of wealth is sufficiently unequal already, then making even more unequal can increase efficiency. Define $M(e)$ as the minimum among of first-period fishing among all Nash equilibria of the game when the distribution of endowments is $e$.

Proposition 4. For all $E$ such that $E>G F$, there exists $\hat{e} \in \Delta(E)$ such that for all mean-preserving spreads $e^{\prime}$ of $\hat{e}, M\left(e^{\prime}\right)<M(\hat{e})$.

Proposition 4 demonstrates that for the wealth distribution $\hat{e}$, where

$$
\hat{e} \equiv\left(E-\frac{G-1}{G} F, \frac{G-1}{G} F\right)
$$


and all mean-preserving spreads of $\hat{e}$, fisher 1 will conserve regardless of the other's behavior. ${ }^{6}$ The proposition also illustrates that the full-conservation equilibrium under perfect inequality in Proposition 3 is a limiting case as inequality is increased. For distributions such as $\hat{e}$, one fisher captures a sufficiently large share of the returns to conservation that he will unilaterally conserve. In particular, there exists an equilibrium in which the larger fisher conserves, the smaller fisher does not, and any mean-preserving spread increases efficiency. If it were true that $i$ 's endowment were greater than $E / G$, then by Proposition 2, fisher $i$ would always conserve if fisher $j$ did. But since $E>(G-1) F$ (which is guaranteed by condition (1)), then $e_{i}<E / G$, and full-capacity period-1 fishing is a best reply by fisher $i$ to full conservation by fisher $j$. Thus any mean-preserving spreads of $\hat{e}$, by further reducing $i$ 's capacity, will increase efficiency, since fisher $j$ will play 0 and more fishing will be deferred until the second period. Thus Olson (1965, p. 35) writes:

This suboptimality or inefficiency will be somewhat less serious in groups composed of members of greatly different size or interest in the collective good. In such unequal groups, on the other hand, there is a tendency toward an arbitrary sharing of the burden of protecting the collective good ... [T] here is accordingly a surprising tendency for the "exploitation" of the great by the small.

This, then, is the commons analogue of the Olson public-goods hypothesis.

\section{[Figure 2 here]}

This situation is summarized in Figure 2, which shows (assuming that $G \geq 2$ ) that as fisher $i$ 's wealth share increases from $\frac{1}{2}$, full efficiency is maintained until his share reaches $(G-1) / G$, at which point the other fisher defects, reducing total catch. Then as the share of $i$ continues to increase, the efficiency of the system increases apace, since the other fisher is capable of harvesting a decreasing fraction of the fish stock in period 1 . When $i$ owns all the wealth, full efficiency is restored. ${ }^{7}$

\footnotetext{
${ }^{6}$ If we restrict the parameters so that $E=F G$, for $G=2$, the wealth distribution $\hat{e}$ is given by $\left(\frac{3}{4} E, \frac{1}{4} E\right)$. As $G$ is increased beyond 2, $\hat{e}$ becomes more unequal.

${ }^{7}$ This figure was suggested by Jean-Marie Baland.
} 


\section{3. $\quad$ Exit options}

In fisheries worldwide, large fishing companies with more opportunities to move their fleets elsewhere (compared to small-scale local fishers) are much less concerned about conservation of the resources in a given harvesting ground. This has been noted in the case of the Texas shrimp fishery by Johnson and Libecap (1982): there, larger fishers have defected from quota schemes. Baland and Plattean (1997) cite a similar phenomenon in a Japanese fishery, where industrial seiners are more apt to deplete fish stocks than local artisanal hook-and-line fishers.

The phenomenon extends to other CPRs. In Mali and Mauritania, large (usually absentee) livestock herd owners have been much less interested than small herders in local arrangements for rangeland management to prevent overgrazing and desertification (Shanmugaranam et al. 1992, cited in Baland and Platteau, 1996). Freudenberger (1991) describes the deforestation of a forest ecosystem in Senegal by the local unit of a nationwide agricultural entity known as the Mouride. A relatively low-intensity pattern of resource use by nearby peasant producers and pastoralists gave way to intensive cash-crop (groundnut) production. After the soil's rapid exhaustion by groundnut farming, the Mouride's national decision-making body could open up new territory elsewhere, unlike traditional users who were more interested in the long-term viability of the local forest. ${ }^{8}$

In all the cases cited above, the richer or larger commons users were prone to defect. This need not always be the case. Other authors have reported that the poorer or smaller users exercise exit options. Bergeret and Ribot (1990), in a study similar to that of Freudenberger, describe deforestation in a larger area and over a longer time frame, also in the Senegalese Sahel. Trees are harvested by Fulani refugees from Guinea, who are more likely to be landless than other peasants, in order to produce charcoal for the rapidly growing urban market. A qualitatively similar situation has been described in southern Burkina Faso, where immigrants are more prone to use destructive gathering techniques in communal forests (Laurent et al. 1994, cited in Baland and Platteau 1997).

\footnotetext{
${ }^{8}$ Ostrom (199) repeatedly stresses the importance of dilemmas like the Mouride case as examples of "heterogeneous discount rates," a form of inequality. If indeed agents have different discount rates, the difference can be explained in terms of deeper parameters, such as different exit possibilities (easily derived from asset inequalities), or other similar factors (e.g., different access to credit).
} 
Let us then augment the basic game presented above so that each fisher has an option to exit rather than fish in the second period. If only one fisher exits in the second period, the other receives the entire second-period catch. Let the value of each fisher i's exit option be given by the function $\psi\left(e_{i}\right)$. This makes the plausible assumption that the exit option depends on a fisher's endowment level: "exit" could refer to investing or deploying one's capacity in another sector. In general, the value of each fisher's exit option will not be the same, unless they have equal endowments. Note that this does not rule out the case where $\psi(\cdot)$ is a constant. It does for the time being rule out the possibility that each fisher has a different exit-option function: that is, we assume that if $e_{i}=e_{j}$, then the fishers' exit options are the same.

When is full conservation an equilibrium in this new setting? For a given fisher $i$, conditional on fisher $j$ 's conservation (that is, $j$ 's first-period catch is zero), it must be that $i$ 's share of the second-period catch is greater than the value of deviating (fishing to full capacity in period 1 and exiting in period 2). That is, for $i=1,2$,

$$
\frac{e_{i}}{E} G F \geq \min \left\{e_{i}, F\right\}+\psi\left(e_{i}\right)
$$

In general, any comparative-static assertions about whether full conservation will be a Nash equilibrium under different wealth distributions will depend on the nature of the $\psi(\cdot)$ function.

3.1. Concave exit options. Thus we will impose the restriction that $\psi\left(e_{i}\right)$ is a concave function, and furthermore that

$$
\psi\left(e_{i}\right) \geq 0
$$

In addition, we restrict attention to cases where "distribution matters"; that is, cases where there exists some distribution such that full conservation is not an equilibrium outcome. This can be stated as follows: there exists some wealth level $e^{\star}, 0<e^{\star} \leq E$, at which

$$
\frac{e^{\star}}{E} G F=\min \left\{e^{\star}, F\right\}+\psi\left(e^{\star}\right)
$$

If assumption (4) is not satisfied, then either full conservation or exit is preferred by both fishers at all levels of wealth, conditional on the conservation of the other. Moreover, for 
simplicity, we shall assume that

$$
\psi(0)=0
$$

Finally, we wish to restrict attention to the case where the fishery is economically viable, in the sense that the maximum possible fish production in the second period is greater than fishing to capacity in the first period and exiting with all of the fishery's capacity in the second period. That is, $G F \geq F+\psi(E)$. This can be restated as:

$$
\psi(E) \leq(G-1) F
$$

In what follows, let the exit strategy be the following course of action by one of the fishers: fish to capacity in period 1 , and exit in period 2. Now we can state the following propositions.

Proposition 5. Consider the augmented game in which each fisher $i$ has a second-period exit option described by the function $\psi\left(e_{i}\right) . \psi(\cdot), G, F$, and $E$ satisfy assumptions (3), (4), and (5). Then, given any wealth distribution $e \in \Delta(E)$ that gives each fisher positive wealth, there exists a mean-preserving spread $e^{\prime}$ of $e$ such that full conservation is not an equilibrium under $e^{\prime}$.

Proposition 5 suggests the following corollary, which addresses the Olson hypothesis in the context of concave exit options.

Corollary 2. If under perfect equality of wealth full conservation is a Nash equilibrium, then there exists a mean-preserving spread $e^{\prime}$ such that full conservation is not an equilibrium.

Corollary 2 says that when the exit option is a nondecreasing concave function, together with the restrictions implied by assumptions (4) and (5), then whenever full conservation is an equilibrium with a perfectly equal distribution of wealth, there always exists a less equal distribution of wealth such that full conservation is not an equilibrium. In this case, equality is more conducive to conservation. Note that under the unequal distribution of 
wealth, it is the poorer agent who finds it in his interest to play the exit strategy. As we will see in a later section, this result generalizes to the case where only one fisher has an exit option.

\section{[Figure 3 here]}

The principal issues raised in Proposition 5 and Corollary 2 can be depicted graphically. First not that the Nash-equilibrium condition (2) can be rewritten as

$$
\psi\left(e_{i}\right) \leq \frac{e_{i}}{E} G F-\min \left\{e_{i}, F\right\}
$$

In Figure 3, fisher $i$ 's wealth is given on the horizontal axis, and $i$ 's payoff is given on the vertical axis. The right-hand side of (7) is drawn as $O N M$, and the left-hand side $(\psi(\cdot))$ is given by the concave function $O S$. Note that the sign of the right-hand side of $(7)$ determines whether full conservation is an equilibrium in the basic (no-exit-option) version of the game introduced in Section 2. From Proposition 2, then, we know that the right-hand side must be positive for all values of $e_{i}$ greater than $E / G$, which is labeled $B$ in Figure 3 . The point labeled $A$ corresponds to $F$, the initial fish stock. $A$ is the point of intersection between the lines $U_{i}=e_{i}\left(\frac{G F}{E}-1\right)$ to the left (note that the slope is negative as a result of the commons dilemma assumption (1)) and $U_{i}=e_{i} \frac{G F}{E}-F$ to the right. The wealth level $e^{\star}$ is labeled $C$. At all wealth levels to the right of $C$, fisher $i$ strictly prefers conservation, conditional on conservation by fisher $j$; at all wealth levels to the left of $C$ (but not including the origin), fisher $i$ prefers the exit strategy.

In Figure 3 and all subsequent figures, $E$ is treated as a constant. That is, as $e_{i}$ is increased, $E$ does not increase; it is assumed that $e_{j}$ is decreased by an equal amount. Alternatively, the horizontal axis of the figures can be interpreted as representing the share of total wealth held by fisher $i$ when $E=1$.

According to Corollary 2, if full conservation is an equilibrium outcome under perfect equality, then there is a mean-preserving spread of the wealth distribution under which full conservation is not an equilibrium. Suppose that the two fishers are initially endowed with wealth $D$ in Figure 3 . Then by redistributing wealth away from fisher $i$ until his wealth lies to the left of $C$, full conservation is no longer an equilibrium; at such a new distribution, $O S$ lies above $O N M$ for fisher $i$, and he will prefer the exit strategy. 


\section{[Figure 4 here]}

Figures 4 and 5 depict the same situation for different forms of the exit-option function. In both figures, the exit options are weakly concave.

In Figure 4, the exit-option function takes the form $\psi\left(e_{i}\right)=b e_{i}$ for nonnegative $b$. It could be that $b$ is an interest rate that can be earned if a fisher's capacity is invested elsewhere. Functions for two values of $b$ are shown. The line $O Q$ represents a value of $b$ so high that neither fisher would ever conserve; this is ruled out by our assumption (4). The line $O P$ represents a lower value of $b$ and can be analyzed like Figure 3. (All points in Figure 4 are labeled as in Figure 3.)

\section{[Figure 5 here]}

Figure 5 depicts an exit-option function of the form

$$
\psi\left(e_{i}\right)= \begin{cases}0, & e_{i}=0 \\ a, & e_{i}>0\end{cases}
$$

for some $a>0$. It can be analyzed in the same way as Figures 3 and 4 . This type of exit optio corresponds to a fixed lump-sum available to each fisher in the second period, and is invariant to their levels of wealth.

Proposition 6 illustrates that the conclusion of Proposition 5 is not completely general; that is, in the vein of Olson, extreme inequality can enhance the prospects for conservation.

Proposition 6. In the exit-option game when assumptions (3) through (6) hold, under perfect inequality $(e=(E, 0)$ or $e=(0, E))$, full conservation is an equilibrium.

Proposition 6 immediately suggests the following corollary.

Corollary 3. For any given distribution of wealth $e$ such that $e_{i}>0$ for $i=1,2$, there always exists a mean-preserving spread of e such that full conservation is an equilibrium. 
Proposition 6 illustrates that the logic of Olson's inequality hypothesis extends to the case of the concave exit option. Proposition 5 illustrates, however, that with concave exit options, Olson's hypothesis cannot be interpreted as a general comparative-static result. Finally, Proposition 7 below indicates that there are cases when the conditions underlying Proposition 5 do not hold: that is, there is no full-conservation equilibrium with perfect equality.

Proposition 7. Consider the game described in Proposition 5, where assumptions (3) through (6) hold. If average wealth is less than $e^{\star}$, then under perfect equality, full conservation is not a Nash equilibrium.

Refer to Figures 3 through 5. Proposition 7 states that if average wealth lies to the left of $C$, then under perfect equality, both fishers would prefer the exit strategy, and full conservation will not be an equilibrium outcome.

As we will see below, this result generalizes to the case where only one fisher has an exit option. Note that the condition in Proposition 7 that average wealth is less than $e^{\star}$ does not contradict assumption (6), which requires only that total wealth is greater than $e^{\star}$. Proposition 7 suggests that whether or not average wealth is less than $e^{\star}$ is an important criterion for whether a given commons situation (with concave exit options) is subject to "Olson effects." Specifically, if average wealth is less than $e^{\star}$, then increased inequality is necessary for equilibrium conservation.

3.2. Convex and asymmetric exit options. In the cases analyzed in Propositions 5 through 7 and illustrated in Figures 3 through 5 , the return to the exit strategy, relative to conservation (and always conditional on conservation by the other fisher) is diminishing in wealth: when there is exit, it is the smaller fisher who exits. In case studies of commons with exit options it is frequently (though by no means exclusively) asserted that, when exit occurs, it is the large resource user who exits. How is the prediction of Proposition 5 reconciled with this empirical evidence? First, it could be that exit options are not concave (or even weakly concave) functions of wealth. Second, it could be that exit option functions 
(and not just the exit-option values) are different for the different fishers. Each of these possibilities is considered in turn.

\section{[Figure 6 here]}

Figure 6 illustrates a convex exit option function. The principal complication is that there are several "crossover" points corresponding to the wealth value $e^{\star}$ in the concave case. Thus, for example, begin at a position of perfect inequality with total wealth $D$ in Figure 6; that is, one fisher's endowment is $D$ and the other's is 0 . Full conservation is not an equilibrium outcome, because the fisher with positive wealth will prefer the exit strategy. If wealth is more equally redistributed in the range of point $C$, full conservation is an equilibrium outcome. If one fisher's wealth is $C$ while the other's is in the range of $A$, however, full conservation is not an equilibrium outcome.

Situations like that depicted in Figure 6 might well describe many commons with exit options. In general, because of the kinked "convex" shape of the right-hand side of the Nash-equilibrium condition (7), a convex left-hand side of the same condition will cross the right-hand side more than once. With convex exit-option functions, we can make the following proposition, which does not, in general, hold when exit-options are concave.

Proposition 8. In the exit-option game where each fisher has an exit option given by $\psi\left(e_{i}\right)$, let $\psi(\cdot)$ be a convex and increasing function of wealth, and let $\psi(0)=0$. If there exists any wealth distribution $e \in \Delta(E)$ such that both fishers have positive wealth and under which full conservation is an equilibrium outcome, then full conservation is not an equilibrium outcome under perfect equality.

In some commons situations, agents' exit options are qualitatively different. In a particular in-shore fishery, for example, it is not simply that the poorer fisher has less capacity, but instead a fundamentally different fishing technology than the larger fisher. The larger fisher can move his ocean-going trawler to another harvesting ground, but if the poor fisher tried to do the same in his small primitive boat, he would stand a good chance of dying at sea. More generally, the smaller agent's capacity is location-specific in a way that the larger agent's is not. 
Suppose that only one fisher has an exit option: this seems a not-too-extreme approximation of the asymmetric-technology argument made in the previous paragraph. Figure 3 can be reinterpreted to depict this case. Suppose that $O S$ is fisher 1's exit-option function, and that $O N M$ is the conditional payoff to conservation for both fishers. Conditional on fisher 1's conservation, fisher 2 will always conserve if his wealth lies anywhere to the right of point $B$. Here the problem is not that fisher 2 will exit, but rather that he will deviate from conservation by fishing to capacity in period 1 . Now if the fishers were to begin at a position of perfect equality at $D$, full conservation would be an equilibrium outcome. If fisher 2's wealth were reduced to some amount between $B$ and $C$ (and fisher 1 's wealth correspondingly increased), full conservation would still be an equilibrium outcome, unlike the situation described by Proposition 5. Nevertheless, if fisher 2's wealth were reduced to a point between $O$ and $B$, he would choose to deviate, and full conservation would not be an equilibrium outcome. If fisher 2's wealth were reduced to $O$, then as in Proposition 6 , full conservation is an equilibrium outcome. Finally, if average wealth lies to the left of $C$, then as in Proposition 7, there is no full-conservation equilibrium under perfect equality.

In the asymmetric exit-option case, our previous interpretation of mean-preserving spreads changes in two ways: first, whether or not a mean-preserving spread destroys a full-conservation equilibrium depends on the identity of the fisher who gains under the redistribution; second, the minimum unequalizing redistribution needed to destroy a fullconservation equilibrium must be more unequalizing than the minimum necessary redistribution in Proposition 5.

Our earlier assumption that exit-option functions were at least weakly concave made strong comparative-static results possible. In the figures, the concavity assumption leads to a sort of "single-crossing" property: there exists a range of wealth levels at which a fisher will not conserve, conditional on the other's conservation, and at all higher wealth levels, the fisher will conditionally conserve. Nevertheless, if there is more than one crossing of the two curves in the diagrams - as in the case of the convex exit-option function - then the comparison of two or more wealth distributions is more complicated. If in the case where there are multiple crossings conservation is not initially an equilibrium outcome, it is not always possible to say whether it will be an equilibrium outcome under any more 
(or less) unequal distribution. If the right-hand side of the inequality (7) is also concave (which might occur under considerably more complicated assumptions about the fishing production function - such as those considered in Section 4.1 below), then even with concave exit-option functions this can give rise to multiple crossings. ${ }^{9}$

The nature of the exit-option functions is ultimately an empirical question. In many situations, exit-option functions are probably linear beyond some level of wealth - this represents a risk-free bond earning a fixed interest rate. But at lower levels of wealth, the exit-option function is convex as a result of borrowing constraints. As we have seen, however, in all cases the presence of exit options generally complicates the prospects for conservation.

\section{Extensions of the Basic modeL}

4.1. Generalizing the catch technology and cost functions. The basic model introduced in Section 2 features a very simple linear production technology and zero cost of effort. In this section we consider a more conventional characterization of costs and production in the fishery. First, there is a cost of supplying fishing effort, and second, the catch per unit of effort is a declining function of aggregate effort, as it becomes more difficult to catch fish as the stock is depleted.

Consider a one-period variant of our basic model. Suppose that fishers choose a level of effort $a_{i}$, as above, but that a fisher's "wealth" is given by the size of his capital stock $k_{i}$, which is fixed each period. Let $A \equiv a_{1}+a_{2}$. Each fisher's catch is a function of individual and aggregate effort:

$$
\phi^{i}\left(a_{i}, A\right)=\Xi(A) a_{i}
$$

and thus aggregate catch $\Phi(\cdot)$ is equal to $\Phi(A)=\Xi(A) A$, where $\Xi^{\prime}(\cdot)<0$ : catch per unit of effort is declining in aggregate effort. Furthermore, suppose that $\Xi^{\prime \prime} \leq 0$ : at higher levels of aggregate effort, the rate at which catch per unit effort declines is greater. Note that this functional form exhibits the congestion externality frequently found in the commons

\footnotetext{
${ }^{9}$ Consistent with the discussion in Bénabou (1996) of "inequality of income versus inequality of power," what matters is not inequality of wealth per se, but inequality of wealth relative to exit options. If the value of one fisher's exit option grows faster than one-for-one with his wealth, then wealth inequality will foster rather than hinder cooperation.
} 
literature (Neher 1990, Mason and Polasky, 1997).

The cost to fisher $i$ is

$$
C\left(a_{i}, k_{i}\right)=c\left(a_{i}, k_{i}\right) a_{i}
$$

where $c_{1}>0$ and $c_{2}<0$ : cost per unit of effort is increasing in effort but decreasing in the capital stock. We also assume that $c_{11} \geq 0$ and that $c_{12} \leq 0$ : as one's capital is increased, the marginal cost of effort decreases.

Thus payoffs are given by $\pi_{i}=\phi_{i}-C(\cdot, \cdot)$, or

$$
\pi_{i}\left(a_{i} ; k_{i}\right)=\Xi(E) e_{i}-c\left(e_{i}, k_{i}\right) e_{i}
$$

Each fisher $i$ seeks to maximize payoff $\pi_{i}$ taking as fixed $a_{j}$ and $k_{i}$. Then using the firstorder conditions from these maximization problems, the Nash equilibrium is the pair of effort levels $\left(a_{1}^{\star}, a_{2}^{\star}\right)$ such that:

$$
\begin{aligned}
& \Xi^{\prime}\left(a_{1}^{\star}+a_{2}^{\star}\right) a_{1}^{\star}+\Xi\left(a_{1}^{\star}+a_{2}^{\star}\right)-a_{1}^{\star} \frac{\partial c}{\partial a_{1}}\left(a_{1}^{\star} ; k_{1}\right)-c\left(a_{1}^{\star} ; k_{1}\right)=0 \\
& \Xi^{\prime}\left(a_{1}^{\star}+a_{2}^{\star}\right) a_{2}^{\star}+\Xi\left(a_{1}^{\star}+a_{2}^{\star}\right)-a_{2}^{\star} \frac{\partial c}{\partial a_{2}}\left(a_{2}^{\star} ; k_{2}\right)-c\left(a_{2}^{\star} ; k_{2}\right)=0
\end{aligned}
$$

We will show that under these conditions, an equalizing redistribution of capital ownership can increase aggregate catch.

Proposition 9. Let

$$
c\left(a_{i} ; k_{i}\right)=\left(\frac{a_{i}}{k_{i}}\right)^{\lambda}
$$

for $i=1,2$ and $\lambda \geq 1$. A mean-preserving spread of the capital distribution $k_{1}=k_{2}=k$ will reduce the aggregate catch.

Proposition 9 shows that increasing inequality reduces aggregate catch. In the static context of this section there is no intertemporal conservation mechanism. By reducing catch, inequality reduces welfare. 
4.2. More than two agents. Most of our results are not qualitatively changed if there are more than two fishers. Proposition 10 extends both our Propositions 2 and 4 . In what follows, we call any outcome in which some but not all fishers fully conserve until the second period "partial conservation." Note that we do not mean that some fishers partially conserve. Say that there is a set of fishers $I$. Although it will not be necessary in the proof of the proposition, let us say that if some subset of fishers $\hat{I} \subset I$ fishes to capacity in period 1 , and they deplete the fish stock, then each of these "defecting" fishers $i$ receives

$$
\frac{e_{i}}{\sum_{i \in \hat{I}} e_{i}} F
$$

Proposition 10. In the basic game with more than two fishers, in which all fishers have positive wealth, partial conservation is an equilibrium outcome if and only if:

1. for all fishers $i$ in the subset $\hat{I} \subset I$ of fishers who do not conserve, $e_{i}<E / G$;

2. for all fishers $i$ in $I \backslash \hat{I}, e_{i} \geq E / G$;

3. $\sum_{i \in \hat{I}} e_{i}<F$

Corollary 4. Suppose that partial conservation is an equilibrium outcome. (a) If there is a fisher $l$ in $I \backslash \hat{I}$ such that $e_{l}>E / G$ and another fisher $m$ in $I \backslash \hat{I}$ such that $e_{m}>e_{l}$, then there exists a mean-preserving spread of the initial wealth distribution under which partial conservation remains an equilibrium outcome. (b) There exist efficiency-enhancing mean-preserving spreads of the initial wealth distribution.

Part (a) of Corollary 4 merely states that mean-preserving spreads of the wealth distribution do not necessarily destroy a cooperative equilibrium outcome: take some wealth from $l$ (but make sure that $e_{l}$ is still greater than or equal to $E / G$ ) and give it to $m$. Part (b) goes further and states that mean-preserving spreads can enhance efficiency in the spirit of Proposition 4 (in the two-fisher case): take some wealth from $p \in \hat{I}$ and transfer it to some $q$ in $I \backslash \hat{I}$ (who is by definition wealthier). Then there is no change in the composition 
of $\hat{I}$, but the amount of first-period fishing is reduced by exactly the amount of wealth taken from $p$.

Finally, if partial conservation is an equilibrium outcome, there exist wealth-equalizing transfers such that full depletion is the only equilibrium outcome under the new wealth distribution. Suppose that $\hat{I}$ is such that $\sum_{i \in \hat{I}} e_{i}=F-\epsilon$ for $\epsilon>0$ small. Then we take wealth

$$
\frac{E}{G}-e_{r}+\epsilon
$$

from some fisher $r \in I \backslash \hat{I}$ and transfer it to some (poorer) fisher $s \in \hat{I}$. Now $r$ will fish to capacity in period 1, but so will all the other fishers, since condition \# 3 of Proposition 10 is no longer satisfied and partial conservation is not an equilibrium outcome.

Now it remains to generalize to the $n$-fisher case Proposition 4, which states that once the wealth distribution is sufficiently unequal, further mean-preserving spreads of that distribution increase equilibrium efficiency. The proof of Proposition 4 constructs this threshold wealth inequality. Proposition 11 below gives sufficient conditions on the wealth distribution such that increases in inequality (weakly) increase equilibrium efficiency in the $n$-fisher extension of the basic game; part of the task of Proposition 11 is to characterize what is meant by "sufficiently unequal" in the many-fisher case.

Let us restrict attention to a particular class of mean-preserving spreads of the wealth distribution. Consider bilateral wealth transfers from a fisher $j$ to a fisher $k$ such that $e_{j}<E / G$ and $e_{k} \geq E / G$. Fisher $j$ would fish to capacity in period 1 before the transfer, and $e_{j} \leq e_{k}$. Call this class of mean-preserving spreads unequalizing wealth transfers. Many more complicated mean-preserving spreads can be characterized as the outcome of a sequence of such unequalizing wealth transfers.

Proposition 11. In the $n$-fisher extension of the basic fishing game, define the set

$$
\bar{I} \equiv\left\{i \in I \mid e_{i} \geq E-F \frac{G-1}{G}\right\}
$$

and define

$$
J \equiv\left\{i \in I \mid e_{i}<\frac{E}{G}\right\}
$$

If $\bar{I}$ and $J$ are nonempty, then after any unequalizing wealth transfer, first-period fishing is weakly lower. 
Proposition 11 states that if there is at least one fisher whose wealth is below the conservation threshold $E / G$, and at least one fisher whose wealth is sufficiently large that he will conserve regardless of the actions of the other fishers, then there always exist wealth redistributions that increase inequality and (at least weakly) equilibrium efficiency.

Note that the conditions of Proposition 11 are not met if all fishers conserve initially (i.e., $J$ is empty). From Proposition 10, we know that this situation can only hold if all fishers have wealth at least as great as $E / G$. Thus in that situation, the wealth distribution is not sufficiently unequal for the Olson-style mechanism of Propositions 4 and 11 to operate.

\section{COnCluding Remarks}

5.1. Crafting distributive rules. In real-world commons problems, economic actors often craft institutions to regulate community use of common-pool resources. If the problem is one of multiple equilibria (as is the case in our model when the conditions of Proposition 2 are satisfied), the task of such local regulation is merely to coordinate actors on one Paretoefficient equilibrium. If the problem is a prisoners dilemma (as is the case under other parameter configurations for our model), there must be a structure of rules, very likely with monitoring and enforcement, that transforms the dilemma into a coordination game and the Pareto-superior outcome into a self-enforcing equilibrium. (This is essentially the message of Ostrom's (1990) synthesis of local regulation of the commons.) Fishers worldwide have elaborated schemes of social regulation with varying degrees of success; many of these cases are reviewed in Baland and Platteau (1998). In this section, we discuss such regulatory regimes in light of our model. ${ }^{10}$

Fishers in our model might consider three regulatory mechanisms to govern the exploitation of the fish stock: they could redistribute wealth $\left(e_{1}, e_{2}\right)$ before period 1 ; they could redistribute catch $\left(\phi_{1}^{1}, \phi_{2}^{1}\right)$ after the first period; or they could redistribute fish $\left(\phi_{1}^{2}, \phi_{2}^{2}\right)$ after the second period. (Many of the distributive rules described in the field-study literature reallocate fishing locations: these can be interpreted as redistributions of capacity. If fishing locations have different productivities, the default share of the fishing stock accruing to each fisher will be different. Note that for our model to apply, it must also be that the fishing

\footnotetext{
${ }^{10}$ The working-paper version of this paper includes a much lengthier analysis of these issues, including the details behind several of the assertions made in the remainder of this section.
} 
locations are not physically isolated from one another.) Such schemes have two possible effects on the payoff of the game. First, the scheme could impose a fine on the player who does not abide by the cooperative agreement: this reduces the return to cheating. Second, output could be shared in the cooperative outcome differently from the default sharing rule of the noncooperative game (i.e., $\left\{e_{i} / E\right\}_{i \in I}$ ). This change in the sharing rule could arise from redistribution of catch, or from pre-play wealth redistribution.

Propositions 1 through 4 and their corollaries are comparative-static results, but can be reinterpreted as statements regarding redistribution of capacity. Thus, for example, Proposition 2 tells us that for wealth distributions that give each fisher positive wealth, full conservation is an equilibrium if and only if each fisher's share of total wealth is greater than $1 / G$. If $G$ is at least two, then there always exists a preplay capacity redistribution such that full conservation is an equilibrium outcome. With the appropriate wealth transfer, full conservation can be supported as an equilibrium, even if it was impossible under the initial distribution. Nevertheless, the magnitude of the transfer under a self-enforcing equilibrium is limited; the fisher who cedes wealth must be at least as well off under full conservation, post-transfer, as under full depletion, pre-transfer.

An alternative to pre-play wealth transfers is that fishers effect transfers of fish conditional on the size of individual first-period catch. Effectively, this means that they can tax each other. It is a well-known result in the fisheries literature that if first-period catch can be taxed at a rate of $100 \%$, then a first-best outcome can be implemented under just about any circumstances (including most exit-option scenarios). (This is essentially the same as boat licensing in our model: limiting the number of boats (i.e., the proportion of $\left.e_{i}\right)$ that each fisher $i$ uses in period 1 is directly related to limiting his catch.) Nevertheless, transaction costs might make it impossible to observe each fisher's period-1 catch and thereby collect taxes. An interesting possibility is that the power to tax is asymmetric; it is plausible to assume that some factor (economic or otherwise) makes it possible for one fisher to impose a sanction on the other, but that the latter is impeded from reciprocating. It can be shown that, under certain conditions, if the poorer fisher is given the power to tax the richer, cooperation is not an equilibrium outcome. This result extends to the case of concave exit options. In the case of convex exit-option functions, however, the poorer fisher 
is better able to enforce conservation.

An interesting consequence of democracy is that it grants to the poor the power to tax the rich. Bardhan (1993) discusses the democratization of environments in which traditional authority structures have previously enforced cooperative agreements. Until democracy is consolidated, cooperative performance of resource users can suffer. ("Resource users" could refer to local villagers sharing a fishery, or to citizens contributing to "social cohesion.") The discussion of asymmetric taxation under exit options shows that this depends on the nature of exit options open to the rich. If the exit-option functions of the rich are convex, then giving the poor the power to tax the rich might not prejudice cooperative behavior. If, however, exit-option functions are concave, cooperation can break down.

Finally, fishers could redistribute the second-period catch once the game is over such that the share accruing to each fisher is a function of his first-period behavior. Assume that the aim of the mechanism is to reduce first-period fishing to zero. If both fish in the first period, both receive their payoffs from the unregulated game $\left(e_{1} F / E, e_{2} F / E\right)$. If both conserve in the first period, then $i$ receives a nonnegative share $\alpha_{i}$ of $G F$, where $\sum \alpha_{i}=1$. If one fisher $i$ cheats in the first period, but the other does not, then $i$ receives some share $\underline{\alpha}_{i}$ of the second-period stock. Effectively, up to this point we have restricted $\alpha_{i}$ and $\left(\underline{\alpha}_{i}\right)$ to equal $e_{i} / E$. It can be shown that under such a rule complex, full conservation can emerge even if $G<n$. However, the range of implementable mechanisms is, under certain circumstances, sensitive to the wealth distribution.

5.2. Summary of results. This paper presents a model of two fishers differentiated by asset-holding levels in an unregulated fishery. Cooperation in this model takes the form of restraint in resource extraction: if both fishers reduce their catch in the first period, they can reap larger rewards after the fish stock has grown. Efficiency is indexed by the amount of the initial fish stock available at the start of the second (and final) period. The model explores the effect of inequality in asset ownership (fishing capacity) on conservation of a commonpool resource. We demonstrate that Olson's (1965) inequality hypothesis - interpreted as a comparative-static statement that increasing inequality enhances efficiency - is not strictly correct. We give conditions such that inequality reduces equilibrium efficiency (conservation of the fish stock). If fishers have earnings opportunities outside the commons 
("exit options") that are concave functions of wealth, increased inequality can reduce the prospects for full conservation. Furthermore, there exists a wealth distribution beyond which increasing wealth inequality increases equilibrium efficiency (though not attaining full conservation as long as both fishers have positive wealth), and full conservation is an equilibrium under perfect inequality. The relationship between inequality and conservation can be U-shaped: at very low and very high levels of inequality, conservation is possible, while for a middle range of inequality, it is not.

\section{Appendix: Proofs of propositions}

Proof of Proposition 1: Suppose fisher 1 plays $e_{1}$. Then fisher 2's choice is between conservation, which yields payoff

$$
\frac{e_{2}}{E} G\left(F-e_{1}\right)
$$

and depletion, yielding

$$
\frac{e_{2}}{E} F
$$

Given that $e_{i}>F(G-1) / G$, then (13) is strictly less than

$$
\frac{e_{2}}{E} G\left(F-F\left(\frac{G-1}{G}\right)\right)=\frac{e_{2}}{E} F
$$

which is (14). Thus fisher 2's payoff to depletion is larger than his payoff to conservation, conditional on fisher 1's having chosen depletion. The argument is the same for fisher 1 . Therefore $\left\{\boldsymbol{e}_{1}, \boldsymbol{e}_{2}\right\}$ is a Nash equilibrium.

Proof of Proposition 2: Suppose that $a_{1}=a_{2}=0$ is an equilibrium. If fisher 1, say, deviates and plays $a>0$ in period 1 , he will receive a total payoff of $a+e_{1} G(F-k) / E$. Since $a_{1}=a_{2}=0$ is a Nash equilibrium, this must be no better than the payoff he would receive under the equilibrium, namely $e_{1} G F / E$. That is,

$$
\begin{array}{r}
e_{1} \frac{G F}{E} \geq a+e_{1} \frac{G(F-a)}{E} \\
e_{1}\left(\frac{G F}{E}-\frac{G(F-a)}{E}\right) \geq a \\
e_{1} \frac{G a}{E} \geq a \Rightarrow e_{1} \geq \frac{E}{G}
\end{array}
$$

Now suppose that $e_{i} \geq E / G$ for $i=1,2$. Suppose that 2 plays 0 and that 0 is not a best reply for 1 . Then there is some $a>0$ such that $a$ is a best reply for 1 . If so, then

$$
e_{1} \frac{G F}{E}<a+e_{1} \frac{G(F-a)}{E}
$$

which implies $e_{1}<E / G$, a contradiction. 
Proof of Proposition 3: The fisher with zero wealth is indifferent between conservation and depletion, given that both yield a payoff of 0 . Conditional on conservation by his opponent, the zero-wealth fisher weakly prefers conservation. The fisher with all the wealth prefers to harvest the fish stock in period 2 (when it is equal to $G F$ ) rather than in period 1 (when it is equal to $F$ ), as long as $G \geq 1$, regardless of his counterpart's action. Thus full conservation is an equilibrium.

Proof of Proposition 4: Let us restrict attention, without loss of generality, to the case where fisher 1 is the larger fisher. Say that fisher 2's endowment is $\epsilon$, and assume furthermore that $\epsilon<F$. If 2 plays his full capacity in period 1, then 1's payoff from full conservation is

$$
\frac{E-\epsilon}{E} G(F-\epsilon)
$$

and his payoff from playing his full capacity in period 1 is

$$
\frac{E-\epsilon}{E} F
$$

The amount (15) is larger than (16) if

$$
\epsilon \leq \frac{G-1}{G} F
$$

Therefore define

$$
\hat{e} \equiv\left(E-\frac{G-1}{G} F, \frac{G-1}{G} F\right)
$$

(Given that $E>F$, this distribution in fact endows fisher 1 more handsomely, as we have assumed.) We have shown so far that full period-1 conservation is always a best reply for fisher 1 to full-capacity fishing by fisher 2 in period 1 . Note that with the distribution given by $\hat{e}, E-\epsilon>E / G$, so that by Proposition 2 , full conservation is also a best reply by fisher 1 to full conservation by fisher 2 . Then for any redistribution of wealth away from fisher 2, fisher 1 will always play 0 in the first period, and thus, regardless of fisher 2's strategy, the amount of fish conserved until the second period will be larger.

Proof of Proposition 5: Assumptions (3), (4) and (5) together imply that condition (2) is satisfied as an equality for fisher $i$ at two points: where $\epsilon_{i}=0$, and where $e_{i}=e^{\star}$ for some $e^{\star}>0$. Moreover, for values of wealth such that $0<e_{i}<e^{\star}$, condition (2) does not hold, while for values of wealth such that $e_{i} \geq e^{\star}$, it does. Consider two cases. (i) Full conservation is an equilibrium outcome under $e$. Then it must be that both fishers hagve wealth greater than $e^{\star}$. Then transfer from one fisher to another an amount such that the first's wealth is now below $e^{\star}$. Then for the first fisher, condition (2) no longer holds, and conservation is no longer an equilibrium outcome. (ii) Full conservation is not an equilibrium under $e$. Then it must be that at least one fisher's wealth lies below $e^{\star}$. Then for any transfer from that fisher to the other, so long as the first still has positive wealth, the wealth distribution will be more unequal, and conservation will not be an equilibrium.

Proof of Proposition 6: Given that the fishery is economically viable (6), the fisher with all the wealth prefers conservation to the exit strategy. The fisher with zero wealth is, by assumption (5), indifferent between conservation and the exit strategy. Therefore full conservation is an equilibrium outcome. 
Proof of Proposition 7: If average wealth is less than $e^{\star}$, then under perfect equality the distribution is given by $(E / 2, E / 2)$ and $e^{\star}>E / 2$. then for neither fisher does condition (2) hold; that is, conditional on the other fisher's conservation, each fisher would prefer the exit strategy. Thus full conservation is not an equilibrium outcome.

Proof of Proposition 8: We will prove the contrapositive of the proposition: that is, we will show that if under perfect inequality, full conservation is not an equilibrium outcome, then there exists no other wealth distribution with $e_{i}>0, \forall i$ under which full conservation is an equilibrium. Suppose that wealth is equally distributed, so that $e=(s, s)$, and that full conservation is not an equilibrium. There are three possible cases. (i) $\psi\left(e_{i}\right)+\min \left\{\epsilon_{i}, F\right\}>\left(\epsilon_{i} / E\right) G F$ for all possible values of $e_{i}, i=1,2$. In this case, both fishers always prefer the exit strategy at all positive values of wealth, so there is no full-conservation equilibrium. Now if the condition $\psi\left(e_{i}\right)+\min \left\{e_{i}, F\right\}>\left(e_{i} / E\right) G F$ is not met, then given the convexity of $\psi(\cdot)$, there is some range of wealth levels over which

$$
\frac{e_{i}}{E} G F \geq \psi\left(e_{i}\right)+\min \left\{\epsilon_{i}, F\right\}
$$

Say that $\underline{e}$ is the lowest value of wealth for which (17) is true, and $\bar{e}$ is the highest level of wealth for which (17) is true. Then if under the distribution $e=(s, s)$, full conservation is not an equilibrium outcome, it must be either that $s<\underline{e}$ or $s>\bar{e}$. These are the two remaining cases we must consider. (ii) $s<\underline{e}$ : All other wealth distributions are mean-preserving spreads of $\epsilon$. If wealth is taken from fisher 1 , say, and given to fisher 2, the latter's wealth could eventually exceed $\underline{e}$, so that 2 would be willing to conserve, conditional on 1's conservation. But 1's wealth will always be less than $\underline{e}$, and given the restriction that both players' wealth always be positive, 1 will for all wealth less than $s$ prefer the exit strategy. So full conservation is not an equilibrium for any wealth distribution other than $e$. (iii) $s>\bar{\epsilon}$ : Then, once again, all other distributions are mean-preserving spreads of $e$. If wealth is given to player 2, the exit strategy will continue to dominate conservation for 2, regardless of 1's strategy. Thus under no mean-preserving spreads of $e$ is conservation an equilibrium outcome.

Proof of Proposition 9: Let the capital distribution be $k_{1}=k_{2}=k$, and consider a mean-preserving spread $\epsilon$ such that the new distribution of capital is $(k+\epsilon, k-\epsilon)$. Then the test is to determine the conditions under which

$$
\frac{d \Phi}{d \epsilon}<0
$$

Note that

$$
\frac{d \Phi}{d \epsilon}=\left(\Xi^{\prime}(\cdot) A+\Xi(\cdot)\right) \frac{d A}{d \epsilon}
$$

The expression in parentheses in (19) is positive. It is the marginal product of (aggregate) effort in equilibrium. The marginal product of aggregate effort is equal to the sum of each fisher's marginal product of individual effort, or the sum of the left-hand sides of the two expressions in (11); this in turn is equal to

$$
\frac{\partial c}{\partial a_{1}}\left(a_{1}^{\star} ; k_{1}\right)+\frac{\partial c}{\partial a_{2}}\left(a_{2}^{\star} ; k_{2}\right)
$$


each term of which is positive for all effort levels by assumption. Thus (18) is true as long as $\frac{d A}{d \epsilon}<0$.

For each fisher $i$, call the first-order conditions in (11) $\Xi^{\prime}(A) a_{i}+\Xi(A)-a_{i} c_{1}^{i}\left(a_{i}, k_{i}\right)-c^{i}\left(a_{i}, k_{i}\right)=0 \equiv$ $F\left(A, a_{i}, k_{i}\right)=0$. Then we can write the Nash-equilibrium action $a_{i}$ as $a_{i}=G\left(k_{i}, A\right)$ for $i=1,2$, and $A=a_{1}+a_{2}=G\left(k_{1}, A\right)+G\left(k_{2}, A\right)$. Then consider the effect of a mean-preserving spread $\epsilon$ :

$$
A=G(k+\epsilon, A)+G(k-\epsilon, A)
$$

Totally differentiating in $(20)$ with respect to $\epsilon$,

$$
d A=\left[G_{1}(k+\epsilon, A)-G_{1}(k-\epsilon, A)\right] d \epsilon+\left[G_{2}(k+\epsilon, A)+G_{2}(k-\epsilon, A)\right] d A
$$

where subscripts on $G$ denote partial derivatives with respect to the numbered argument. Thus

$$
\frac{d A}{d \epsilon}=\frac{G_{1}(k+\epsilon, A)-G_{1}(k-\epsilon, A)}{1-\left(G_{2}(k+\epsilon, A)+G_{2}(k-\epsilon, A)\right)}
$$

First we shall show that $a_{i}$ is an increasing and concave function of $k_{i}$, holding $A$ constant. Implicitly differentiating the first-order conditions for Nash equilibrium (11) (holding $A$ constant) and rearranging,

$$
\left.\frac{d a_{i}}{d k_{i}}\right|_{d A=0}=\frac{a_{I}\left|\frac{\partial^{2}}{\partial a_{i} \partial k_{i}}\left(a_{i} ; k_{i}\right)\right|+\left|\frac{\partial c}{\partial k_{i}}\left(a_{i} ; k_{i}\right)\right|}{\left|\Xi^{\prime}(A)\right|+2 \frac{\partial c}{\partial a_{i}}\left(a_{i} ; k_{i}\right)+a_{i} \frac{\partial^{2}}{\partial a_{i}^{2}}\left(a_{i} ; k_{i}\right)}>0
$$

With the specific form of the $c(\cdot ; \cdot)$ function given in the proposition, (22) is equivalent to:

$$
\frac{(1+\lambda) \frac{c\left(a_{i} ; k_{i}\right)}{k_{i}}}{\frac{\left|\Xi^{\prime}(A)\right|}{\lambda}+\frac{c\left(a_{i} ; k_{i}\right)}{a_{i}}(1+\lambda)}
$$

Then it can easily be shown that

$$
\left.\frac{d^{2} a_{i}}{d k_{i}^{2}}\right|_{d A=0}<0
$$

Now we show that in (21), $G_{2}$ is negative. Again using the first-order conditions (11),

$$
G_{2}=\left.\frac{d a_{i}}{d A}\right|_{d k_{i}=0}=\frac{\Xi^{\prime \prime}(A) a_{i}+\Xi^{\prime}(A)}{\left|\Xi^{\prime}(A)\right|+2 \frac{\partial c}{\partial a_{i}}\left(a_{i} ; k_{i}\right)+a_{i} \frac{\partial^{2}}{\partial a_{i}^{2}}\left(a_{i} ; k_{i}\right)}>0
$$

As $G_{2}<0$, the denominator in (21) is positive. As $a_{i}$ is concave in $k_{i}$, we can say that

$$
G_{1}(k+\epsilon, A)<G_{1}(k-\epsilon, A)
$$

Therefore, from (21),

$$
\frac{d A}{d \epsilon}<0
$$

This establishes (18).

Proof of Proposition 10 (sketch): The proof is very simple and will not be given in full. If condition \# 3 of the proposition is satisfied, then the depleting coalition leaves some fish to regenerate between periods; if \# 3 is not satisfied, then the only equilibrium outcome is full depletion in period 1. Say that the fishers in $\hat{I} \subset I$ fish to capacity in period 1 , and condition \# 3 is satisfied. Then a fisher $j$ not in $\hat{I}$ gets payoff

$$
\frac{e_{j}}{E} G\left(F-\sum_{i \in \hat{I}} e_{i}\right)
$$


from conserving, and fishing to capacity in period 1 yields him

$$
\frac{e_{j}}{E} G\left(F-\sum_{i \in \hat{I}} e_{i}-e_{j}\right)+\epsilon_{j}
$$

Now (23) is at least as large as (24) if and only if $e_{j} \geq E / G$. By similar logic, if a fisher $k$ nominally in $\hat{I}$ is unilaterally deciding between conserving or depleting, the condition for staying in $\hat{I}$ is that $e_{k}<E / G$.

Proof of Proposition 11: In each case that follows, consider a transfer from fisher $j$ to fisher $k$. If $k \in \bar{I}$, then $k$ will always conserve regardless of the choices made by other fishers. To see this, consider $k$ 's choice. Say that all other fishers fish to capacity in period 1 , and that $E_{-k} \equiv \sum_{i \neq k} e_{i}$ is the sum of wealth held by all other fishers. Furthermore, assume that $E_{-k}<F$. Then if $k$ chooses to conserve, his payoff is

$$
\frac{e_{k}}{E} G\left(F-E_{-k}\right)
$$

while if $k$ fishes to capacity in period 1 , his payoff is $\left(e_{k} / E\right) F$. Now $(25)$ is at least as large as $\left(e_{k} / E\right) F$ if

$$
E_{-k} \leq \frac{F(G-1)}{G}
$$

now since $e_{k}=E-E_{-k},(26)$ is equivalent to

$$
e_{k}=E-E_{-k} \geq E-\frac{F(G-1)}{G}
$$

But (27) always holds by $k$ 's inclusion in $\bar{I}$. Thus for any fisher with wealth sufficiently great to be in $\bar{I}$ conservation is a dominant strategy.

Now consider an unequalizing wealth transfer to such a fisher $k$. The result of such a transfer is that the fisher $j$ who loses wealth must reduce first-period fishing one-for-one with his wealth reduction; $k$ waits to deploy his wealth, including the transfer from $j$, until the second period. Thus period-1 fishing is strictly decreased and equilibrium efficiency is strictly increased.

We must also consider unequalizing transfers $j \in J$ to fishers $k$ not in $\bar{l}$. If $k \in J$, then $e_{k}<E / G$. After the transfer $\eta>0, j$ 's period-1 fishing is decreased by $\eta$. If $\epsilon_{k}+\eta \geq E / G$, then $k$ could choose to conserve and equilibrium efficiency would strictly increase. If $e_{k}+\eta<E / G, k$ will increase his period-1 fishing by $\eta$ and aggregate period-1 fishing (and thus equilibrium efficiency) is unchanged. Suppose finally that $k$ is neither in $J$ nor $\bar{l}$. As before $j$ will decrease his period-1 fishing by $\eta$ after the transfer. If $k$ conserves in equilibrium before the transfer, $k$ will continue to do so after the transfer; aggregate period-1 fishing is decreased by $\eta$. Even if $k$ did not conserve before the transfer, he could now find it optimal to do so, and equilibrium efficiency would increase. If $k$ did not conserve before the transfer, and still chooses not to after the transfer, his increased fishing exactly offsets $j$ 's reduction and equilibrium efficiency is unchanged.

\section{ACKNOWLEDGM ENTS}


We thank Jean-Marie Baland, Roland Bénabou, Tim Besley, Sam Bowles, Chuck Clarke, and especially Matthew Rabin for comments. Seminar participants at the University of California, Berkeley, the Universidad de Guanajuato, and a meeting ofthe MacArthur Foundation's research network on Inequality and Economic Performance provided valuable feedback. Financial support from the MacArthur Foundation is gratefully acknowledged. An earlier, somewhat different, version of this paper circulated as Center for International and Development Economic Research (CIDER) Working Paper No. 096-071, University of California, Berkeley.

\section{REFERENCES}

[1] Baland, J.-M., Platteau, J.-P., 1996. Halting degradation of natural resources: Is there a role for rural communities? Oxford University Press, Oxford.

[2] Baland, J.-M., Platteau, J.-P., 1997. Wealth inequality and efficiency in the commons, i: The unregulated case. Oxford Economic Papers 49, 451-482.

[3] Baland, J.-M., Platteau, J.-P., 1998. Wealth inequality and efficiency in the commons, ii: The regulated case. Oxford Economic Papers 50, 1-22.

[4] Bardhan, P. K., 1993. Symposium on management of local commons. Journal of Economic Perspectives $7,87-92$.

[5] Bardhan, P. K., 1995. Rational fools and cooperation in a poor hydraulic economy. In: Basu, K., Pattanaik, P., and Suzumura, K. (Eds.) Choice, welfare, and development: A Festchrift in honour of Amartya K. Sen. Clarendon Press, Oxford.

[6] Bénabou, R., 1996. Inequality and growth. NBER Macroeconomics Annual 11, 11-74.

[7] Benhabib, J., Rustichini, A., 1996. Social conflict and growth. Journal of Economic Growth 1, $125-142$.

[8] Bergeret, A., Ribot, J. C., 1990. L'arbre nourricier en pays sahelien. Editions de la Maison des sciences de l'homme, Paris.

[9] Bergstrom, T. C., Blume, L., Varian, H., 1986. On the private provision of public goods. Journal of Public Economics 29, 25-49.

[10] Chan, K. S., Mestelman, S., Moir, R., Muller, R. A., 1996. The voluntary provision of public goods under varying income distributions, Canadian Journal of Economics 19, 54-69.

[11] Dutta, P., Sundaram, R. K., 1993. The tragedy of the commons? A characterization of stationary perfect equilibrium in dynamic games, Economic Theory 3.

[12] Freudenberger, K. S., 1991. Mbegué: The disingenuous destruction of a Sahelian rainforest. International Institute for Environment and Development Paper No. 29, London. 
[13] Government of Canada, 1999. Final Report on Social Cohesion. Standing Senate Committee on Social Affairs, Science and Technology, Ottawa.

[14] Johnson, R. N., Libecap, G. D., 1982. Contracting problems and regulation: The case of the fishery. American Economic Review 72, 1005-1022.

[15] Kanbur, R., 1991. Heterogeneity, distribution and cooperation in common property resource management. Background paper for the 1992 World Development Report, The World Bank, Washington D.C.

[16] Keohane, R. O., Ostrom, E., 1994. Introduction to the symposium "Local commons and global interdependence: Heterogeneity and cooperation in two domains." Journal of Theoretical Politics 6, 403-428.

[17] Knack, S., Keefer, P., 1997. Does social capital have an economic payoff? Quarterly Journal of Economics $112,1251-1332$.

[18] Laurent, P. J., Mathieu, P., Totté, M., 1994. Migrations et accès à la terre au Burkina Faso. Cahiers du CIDEP 20.

[19] Levhari, D., Mirman, L. J., 1980. The great fish war. Bell Journal of Economics 11, 322-44.

[20] Mason, C.F., Polasky, S., 1997. The optimal number of firms in the commons: A dynamic approach. Canadian Journal of Economics 30, 1143-1160.

[21] Neher, P., 1990. Natural resource economics: Conservation and exploitation. Cambridge University Press, Cambridge, U.K.

[22] Olson, M., 1965. The logic of collective action. Harvard University Press, Cambridge, Mass.

[23] Ostrom, E., 1990. Governing the commons: The evolution of institutions for collective action. Cambridge University Press, New York.

[24] Safina, C., 1995. The world's imperiled fish. Scientific American 273, 46-53.

[25] Shanmugaranam, N., Vedeld, T., Mossige, A., Bovin, M., 1992. Resource management and pastoral institution-building in the West African Sahel. Discussion Paper No. 175, The World Bank, Washington, D.C.

[26] Tornell, A., Velasco, A., 1992. The tragedy of the commons and economic growth: Why does capital flow from poor to rich countries? Journal of Political Economy 100, 1208-1231. 